\title{
Comparison of fuel consumption and exhaust emissions in WLTP and NEDC procedures
}

Fuel consumption achieved in the New European Driving Cycle (NEDC) could be 50\% lower than the fuel consumption in real driving conditions and in the case of emissions of regulated toxic compounds the differences could even be much greater. In order to bring the results achieved in official tests closer to real life figures, the European Commission introduced in 2017 the Worldwide Harmonized Light Vehicles Test Procedure (WLTP), which replaced the NEDC. In this article the results of fuel consumption and exhaust emissions for 3 cars fitted with engines of the same displacement but with direct and indirect gasoline injection, determined according to the NEDC and WLTC were presented. The results show that the effect of driving cycle on the fuel consumption is equivocal - for one car, fuel consumption was higher in the WLTC; for the other one in the NEDC; and for the third one, fuel consumption achieved in both driving cycles was practically the same. Emissions of regulated exhaust compounds, except for THC, obtained in the WLTC were higher than in the NEDC driving cycle.

Key words: exhaust emissions, fuel consumption, driving cycle, type-approval, chassis dynamometer

\section{Introduction}

Determination of fuel consumption and emissions of toxic compounds of passenger vehicles is important from points of view of environment protection and prospective car buyers. Fuel consumption achieved in the New European Driving Cycle (NEDC) sometimes could be up to $50 \%$ lower than the fuel consumption in real driving conditions, and in the case of emissions of regulated toxic compounds the differences could be even much greater. It is estimated that on average the divergence between type-approval based on the NEDC and real-world fuel consumption of new passenger cars in Europe was increasing over time, from $8 \%$ in 2001 to $40 \%$ in 2014 [6]. On road emission of $\mathrm{NO}_{\mathrm{x}}$ was estimated to be on average 6-7 times higher than the values measured in the NEDC [9].

The divergence of type-approval and real-world values varied by segment, manufacturer, fuel, transmission and so on. Generally that divergence was greater for passenger vehicles with diesel engines than with spark ignition (SI) engines $[1,4,6]$.

The reason for the above discrepancies were different conditions of vehicle operation during official testing in the laboratory according to the NEDC and during real vehicle use on the road. Those different conditions resulted of different driving cycle (speed profile, gear shift points) and vehicle configuration (mass, equipment, pressure and type of tires and so on).

In order to bring closer the results achieved in official tests to real life figures, the European Commission introduced in 2017 the Worldwide Harmonized Light Vehicles Test Procedure (WLTP), which replaced the NEDC. The new procedure introduces a more dynamic World Harmonized Light Duty Vehicle Test Cycle (WLTC) and other changes in testing conditions, including various vehicle configurations.

The effect of replacing the NEDC with the WLTP was evaluated in many studies. The $\mathrm{CO}_{2}$ emission is on average $11 \%$ higher for the WLTP worst case scenario (highest vehicle mass and highest rolling resistance), and $1 \%$ higher for the WLTP best case scenario (smallest vehicle mass and rolling resistance), in comparison to the emissions achieved in the NEDC [5]. Results from this study also show that moving from NEDC to WLTP has a stronger impact on diesel than on gasoline vehicles.

On the other hand, the estimations presented in [2] indicate that fuel consumption and $\mathrm{CO}_{2}$ emission in WLTP tests are on average $6 \%$ lower than in NEDC tests (in the case of SI engines $-10 \%)$. $\mathrm{NO}_{\mathrm{x}}$ emission is $1 \%$ higher in WLTP cycle, comparing with NEDC, however for SI vehicles only, it is lower by $16 \%$. Estimated HC emission for gasoline vehicles is $26 \%$ lower in WLTP than in NEDC.

Simulations presented in [7] indicate that the $\mathrm{CO}_{2}$ emission in WLTP will be by $20 \mathrm{~g} / \mathrm{km}$ greater than in NEDC for the fleet of passenger cars. According to [3] this increase will be equal to about $26 \mathrm{~g} / \mathrm{km}$, but the gap between realword and WLTP $\mathrm{CO}_{2}$ emissions will still remain, although will be smaller. These statements are only partially confirmed by the results presented in [8]. In the case of vehicles with diesel engines $\mathrm{CO}_{2}$ emissions achieved in RDE (real driving emission) tests were by $6 \%$ higher than achieved in WLTP tests. However, in the case of vehicles with SI engines the $\mathrm{CO}_{2}$ emissions were $2 \%$ lower. $\mathrm{NO}_{\mathrm{x}}$ emissions in RDE tests were higher by $20 \%$ for vehicles with diesel engine, and by $6 \%$ for vehicles with SI engines. At the same time, the authors emphasize the great impact of testing conditions (cold operation, road grade, trip selection and driving style) on results achieved in RDE tests.

Summarizing, the influence of the new testing procedure (WLTP) on the emissions is not obvious, especially in the case of vehicles with SI engines. To evaluate the effect of the driving cycle on fuel consumption and exhaust emissions, 3 cars fitted with SI engines were tested using the NEDC and WLPC cycles. It should be underlined that driving cycle is a relevant part of the test procedure, but it is not the only important factor. The method and parameters used for simulation of vehicle mass and road load are pretty important. The ambient conditions, i.e. temperature, also exert an influence on the results. This paper focuses on 
differences caused by the driving cycles themselves, and so the broader test procedures is not examined and discussed in detail.

\section{Objects and method}

\subsection{Vehicles tested}

Two of tested vehicles were mini cars (segment A) and one a medium car (segment $\mathrm{C}$ ). The distances covered by the cars before the tests were different: one of them was almost new, just well run-in $(2000 \mathrm{~km})$, the second had mileage of $20000 \mathrm{~km}$ and the third of $60000 \mathrm{~km}$. All of the cars tested were in a very good technical state. All cars were equipped with manual gearboxes and four-cylinder, spark ignition engines of the same displacement of $1.4 \mathrm{dm}^{3}$. One of the engines was naturally aspirated and two were turbocharged, and one engine had direct gasoline injection and two indirect multipoint injection system. A detailed specification of the tested vehicles and engines is presented in Table 1.

Table 1. Specification of tested vehicles

\begin{tabular}{|l|c|c|c|}
\hline Parameter & Vehicle A & Vehicle B & Vehicle C \\
\hline Vehicle category & M1 & M1 & M1 \\
\hline Vehicle segment ${ }^{*}$ & $\mathrm{C}$ & $\mathrm{A}$ & $\mathrm{A}$ \\
\hline Vehicle mass $[\mathrm{kg}]$ & 1360 & 1160 & 1130 \\
\hline Vehicle mileage $[\mathrm{km}]$ & 60000 & 20000 & 2000 \\
\hline Engine type & $\mathrm{SI} / \mathrm{R} 4$ & $\mathrm{SI} / \mathrm{R} 4$ & $\mathrm{SI} / \mathrm{R} 4$ \\
\hline Swept volume $\left[\mathrm{dm}^{3}\right]$ & 1.4 & 1.4 & 1.4 \\
\hline Injection / charging & DI / turbo & MPI / turbo & $\mathrm{MPI} /-$ \\
\hline Engine power [kW] & 90 & 107 & 57 \\
\hline \multicolumn{4}{|l}{ A - mini cars, C-medium cars } \\
\hline
\end{tabular}

\subsection{Laboratory}

Tests were carried out in BOSMAL's exhaust emissions laboratory. This climate controlled test facility meets all the demands of Euro 5/6 and SULEV, as well as the stipulations of GTR 15. The vehicles were tested on a chassis dynamometer AVL Zöllner 48”. Emissions measurement and analysis was performed using the laboratory's Horiba CVS-CFV sampling system, together with a dilution tunnel Horiba DLT-7020 and a set of Horiba MEXA-7400HRTLE exhaust analyzers for simultaneous measurement of concentrations of carbon monoxide, carbon dioxide, hydrocarbons and oxides of nitrogen. Particle measurement was performed using system HORIBA DLS 7100E and particle counter HORIBA MEXA 2000 SPCS. All the aforementioned devices were controlled by the Horiba VETS7000NT management system. Sample bags for the sampling of ambient air and exhaust gas were made of Tedlar ${ }^{\mathrm{TM}}$ and housed in a heated, insulated unit maintained at $35^{\circ} \mathrm{C}$ to prevent condensation.

\subsection{Procedure}

During the tests the tested vehicle were driven over two test cycles: NEDC and WLPC (Fig. 1).

The NEDC test cycle consists of two phases. The first of them simulates urban driving and contains 4 times repeated ECE 15 Urban Driving Cycles (UDC). The second phase - Extra-Urban Driving Cycle (EUDC) reflects highway driving with maximum speed of $120 \mathrm{~km} / \mathrm{h}$. The total duration of the cycle is $1180 \mathrm{~s}$, theoretical distance is 10.93 $\mathrm{km}$, and average speed is $33.35 \mathrm{~km} / \mathrm{h}(43.10 \mathrm{~km} / \mathrm{h}$ excluding stops, which last $267 \mathrm{~s}$ ). In the NEDC test the speed trace and gearshift timing are fixed and the same for all kind of cars.

The WLTC test cycle is the new speed trace of the new World Harmonized Light Vehicles Test Procedure. According to WLTP, there are 3 WLTC driving cycles: class 1,2 and 3 . The class is chosen taking into account engine power to vehicle mass ratio. All 3 examined vehicles, as practically all European passenger cars, belong to class 3 (max power/kerb weight $>34 \mathrm{~W} / \mathrm{kg}$ ). WLTC class 3 cycle consists of 4 phases: low, medium, high and extra high which simulate successively urban, extra urban and highway driving with max speed of $131.3 \mathrm{~km} / \mathrm{h}$. The whole test lasts $1800 \mathrm{~s}$ and the vehicle travels a distance of $23.27 \mathrm{~km}$ at an average speed of $46.5 \mathrm{~km} / \mathrm{h}(53.5 \mathrm{~km} / \mathrm{h}$ excluding stops, which last $235 \mathrm{~s}$ in total) during this time. The gearshift timing in WLTC is not fixed, but is calculated by a specific computer algorithm for every vehicle taking into account vehicle weight and engine full load power characteristic.

Tests were carried out according to the test procedure applicable to the test cycle in question, but the basic approach was the same. For the vehicles tested in this work, the prescribed gearshifts were used for the NEDC and the calculated gearshifts for the WLTC (different for each vehicle tested). Dynamometer settings has been determined using the alternative methods according to the applicable regulations. However, the vehicle configuration (mass, tire type and pressure, fuel) was the same in NEDC and WLTC tests.

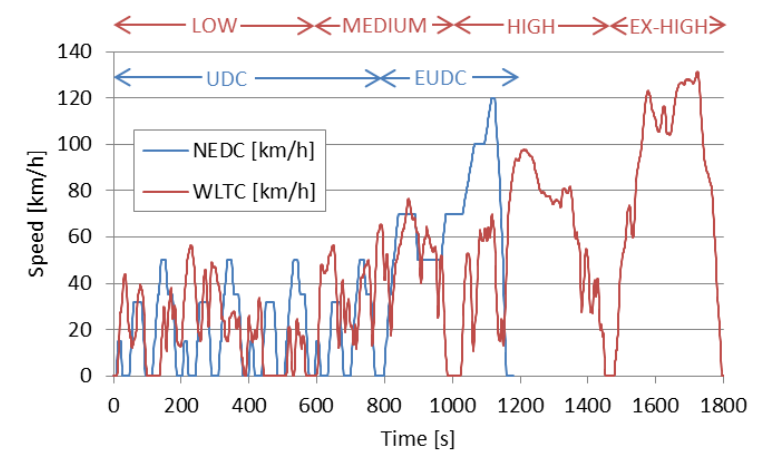

Fig. 1. Speed profile for WLTC and NEDC cycle

\section{Results and discussion}

\subsection{NEDC}

Fuel consumption (FC) and emissions of carbon monoxide (CO), unburned hydrocarbons (THC), nitrogen oxides $\left(\mathrm{NO}_{\mathrm{x}}\right)$ and particle number $(\mathrm{PN})$ obtained in each phase of the NEDC and in the entire cycle for the tested vehicles are presented in Figs 2-6. As expected, the lightest car equipped with the least powerful engine (vehicle C) had the smallest fuel consumption in the NEDC cycle, however the highest fuel consumption had a vehicle B of almost the same mass but equipped with the most powerful engine. Fuel consumption of considerably heavier vehicle A was by $4 \%$ lower than fuel consumption of vehicle $\mathrm{B}$, and $9 \%$ greater than of vehicle $\mathrm{C}$. For all cars fuel consumption in urban phase (UDC) was much greater than in highway phase (EUDC) - from almost 50\% for vehicle A to over 
$80 \%$ for vehicle B. Despite of the heaviest mass vehicle A had only $3 \%$ greater fuel consumption than vehicle $\mathrm{C}$ and $16 \%$ smaller than vehicle B during UDC. On the other hand in spite of the 6-speed gearbox vehicle A had the highest fuel consumption in EUDC (vehicles $\mathrm{B}$ and $\mathrm{C}$ were equipped with 5-speed gearboxes).

Emissions of toxic compounds during NEDC cycle from particular vehicle tested differ significantly, but it is hard to point at the vehicle with the lowest or highest emission, because it depends on the compound considered (Figs 3-6). Generally, for each of the vehicles, emissions of all toxic compounds are much higher in UDC than during EUDC, what is related to the lower average speed - 18.4 $\mathrm{km} / \mathrm{h}$ in UDC vs $62.6 \mathrm{~km} / \mathrm{h}$ in EUDC, and cold engine at the beginning of the UDC. Particularly large differences between results in UDC and EUDC occurred in the case of hydrocarbons, where the THC emission in UDC was at least 50 times higher than in the EUDC for each of the tested cars (Fig. 4). As expected, the emission of particulate matter from the vehicle equipped with a direct injection engine (vehicle A) was higher than that of cars with indirect injection engines (Fig. 6).

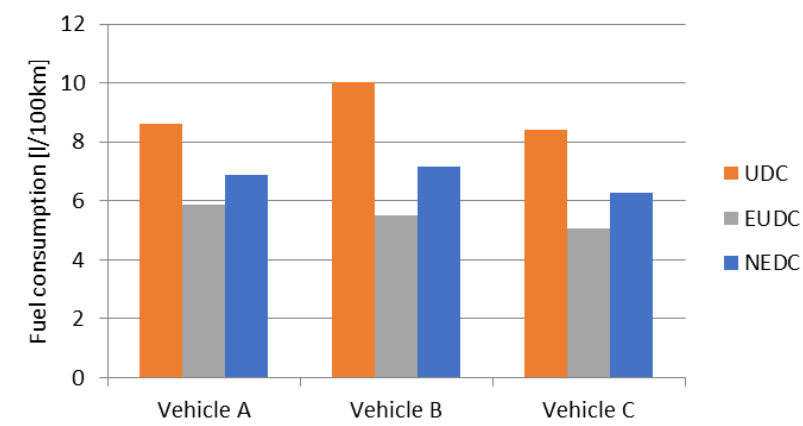

Fig. 2. Fuel consumption in NEDC test and its particular stages

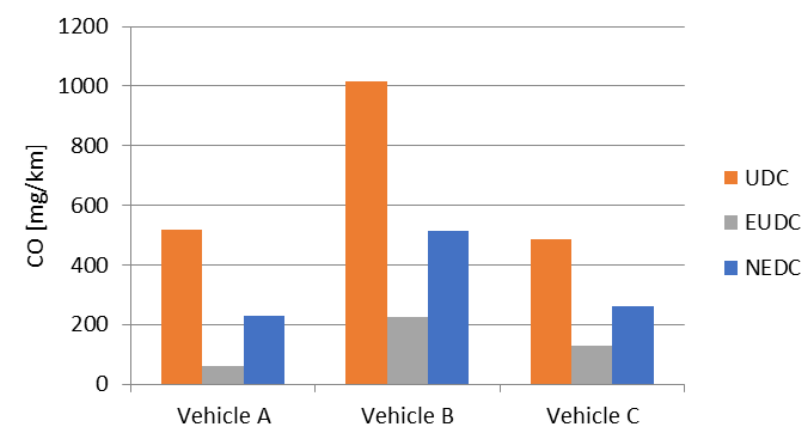

Fig. 3. Carbon monoxide emissions in NEDC test and its particular stages

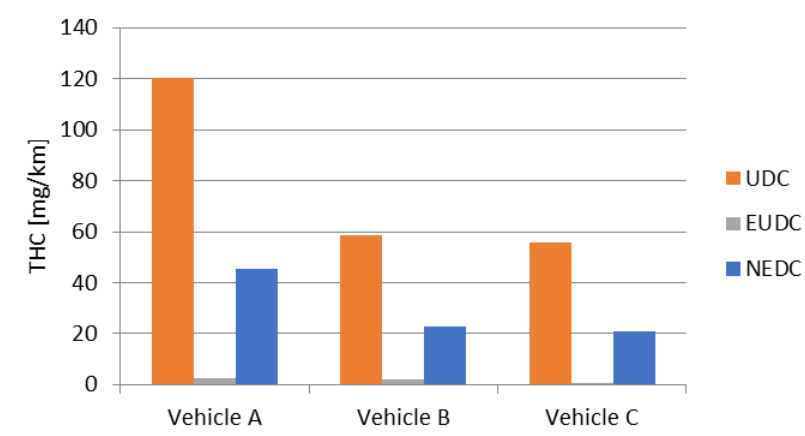

Fig. 4. Hydrocarbons emissions in NEDC test and its particular stages

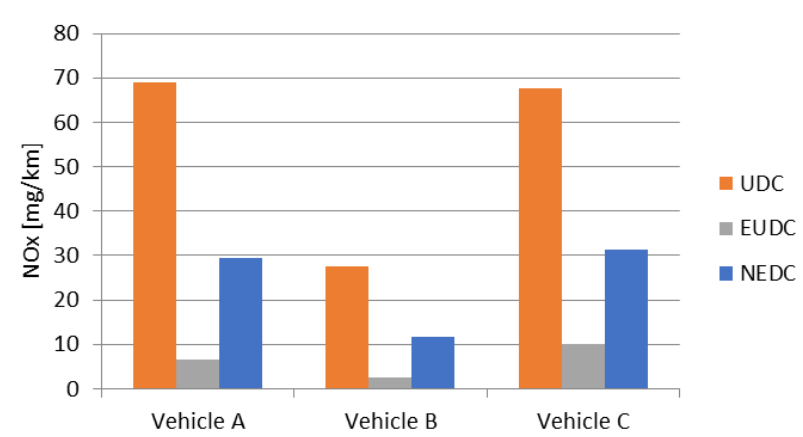

Fig. 5. Nitrogen oxides emissions in NEDC test and its particular stages

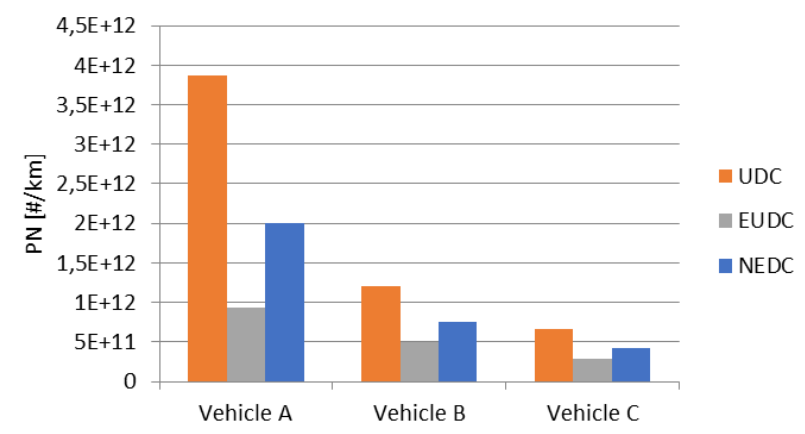

Fig. 6. Particle emissions in NEDC test and its particular stages

\subsection{WLTC}

Fuel consumption in the WLTC cycle and in its particular stages for all tested vehicle are presented in Fig. 7. The heaviest of the cars (vehicle A) consumed the most fuel, and the lightest one the least (vehicle C). Such trend was also observed for particular phases of the cycle, apart from the low phase, where vehicle B consumed the most fuel. It should be also noted that despite of the 6-speed gearbox vehicle A had much greater fuel consumption than the other cars in extra high phase. All tested vehicles achieved the highest fuel consumption in the first phase of the WLTC, and the lowest in the third.

The emissions of toxic compounds in WLTC cycle and its particular phases are presented in Figs 8-11. As in the NEDC test, it is difficult to identify the vehicle with the highest or lowest emission in the WLPC cycle. Vehicle A had the highest particulate emission, vehicle $\mathrm{B}$ the highest $\mathrm{CO}$ emissions, and vehicle $\mathrm{C}$ the highest NOx emission.

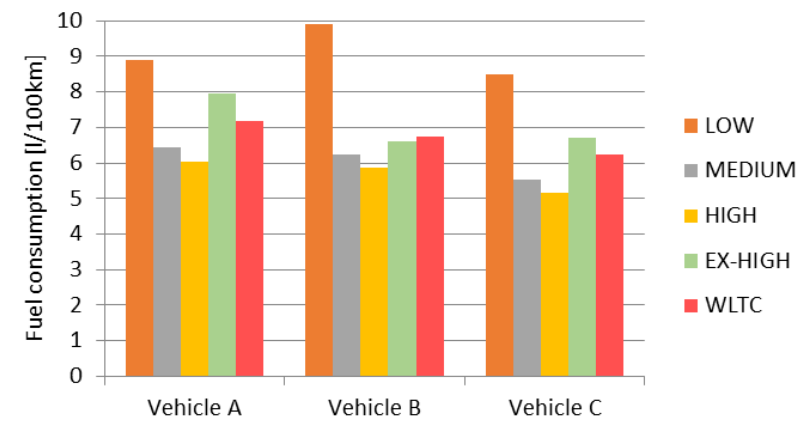

Fig. 7. Fuel consumption in WLTC test and its particular stages

For all cars tested, the emission of all toxic compounds in the first phase of the WLTC was definitely higher than in the remaining ones. The greatest difference, as in the 
NEDC, was in the case of hydrocarbons. This was caused by long stop times ( $27 \%$ of the duration of the phase) and low average speed $(19 \mathrm{~km} / \mathrm{h})$ in this phase, and above all by the heating up of the engines and aftertreatment systems.

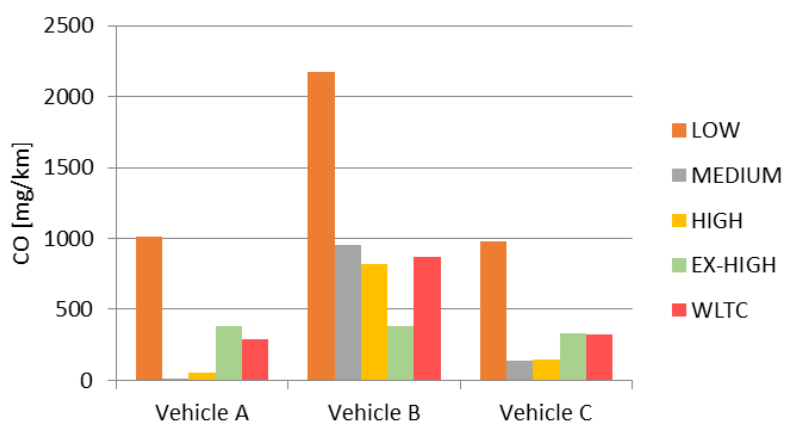

Fig. 8. Carbon monoxide emissions in WLTC test and its particular stages

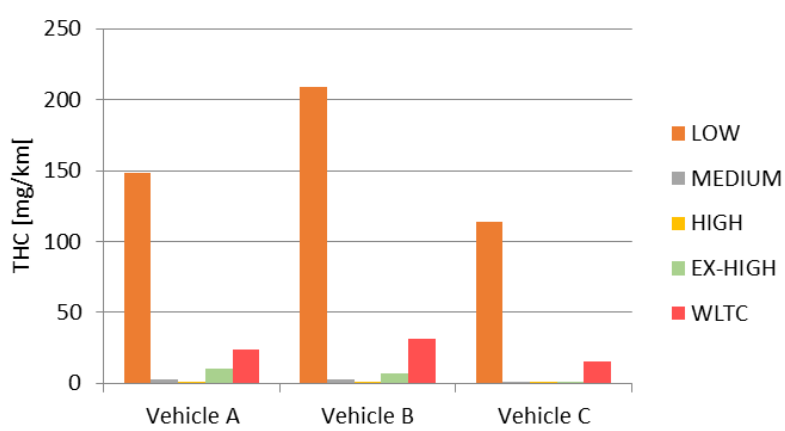

Fig. 9. Hydrocarbons emissions in WLTC test and its particular stages

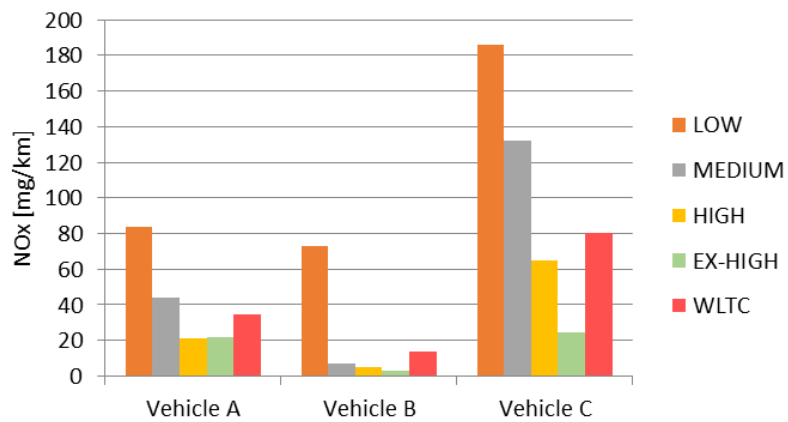

Fig. 10. Nitrogen oxides emissions in WLTC test and its particular stages

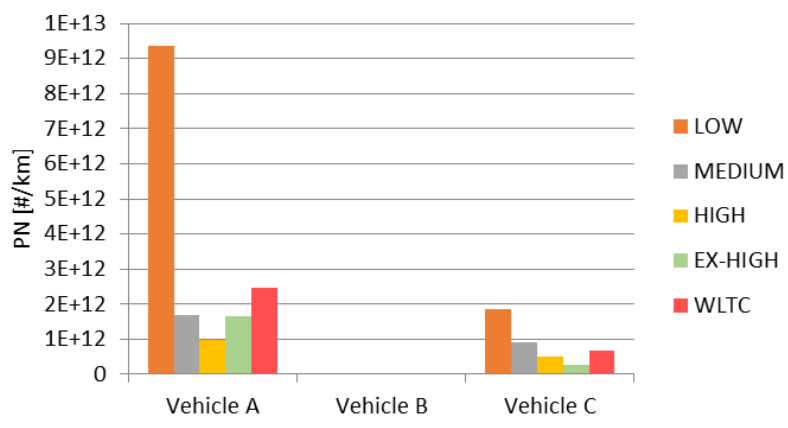

Fig. 11. Particle emissions in WLTC test and its particular stages

\subsection{Comparison of results from NEDC and WLTC}

The results achieved in the NEDC and WLTC cycles are compared in Figs 12-16. Only vehicle A achieved higher fuel consumption in the WLTC cycle than in the NEDC cycle (by $4.5 \%$ ). The other two cars had lower fuel consumption in the WLTP cycle - vehicle B by $6 \%$ and vehicle C by $1 \%$ (Fig. 12).

The emissions of carbon monoxide (CO), nitrogen oxides $\left(\mathrm{NO}_{\mathrm{x}}\right)$ and particulate number $(\mathrm{PN})$ were higher in the WLTC than in the NEDC, by $27 \%, 70 \%$ and $24 \%, 17 \%$, $19 \%$ and $156 \%$, and $23 \%$, undetermined and $64 \%$, respectively for vehicles A, B and C (Figs 13, 15 and 16). Only vehicle B had lower hydrocarbons (THC) emission in the WLTC, by $53 \%$ in comparison to THC emission in the NEDC. THC emissions for the other two cars in the WLTC were lower by $47 \%$ and $24 \%$, respectively for vehicle A and $\mathrm{C}$ (Fig. 14). The effect of the cycle on particulate matter emission (PM) was determined only for vehicle A and this emission was greater by $10 \%$ in the WLTC (Fig. 16). PMs were measured only for entire cycles, not for their particular phases.

It should be noted that all tested cars in both NEDC and WLTC cycles met the requirements of Euro 6 standard for CO, THC and PM emissions - the limits are $1000 \mathrm{mg} / \mathrm{km}$, $100 \mathrm{mg} / \mathrm{km}$ and 4,5 mg/km, respectively. In the case of $\mathrm{NO}_{\mathrm{x}}$ emission, only vehicle $\mathrm{C}$ in the WLTC did not fulfill the requirement - the limit is $60 \mathrm{mg} / \mathrm{km}$ (Fig. 15). The Euro 6 PN emission limit of $6 \times 10^{11} \# / \mathrm{km}$ has been significantly exceeded by vehicle A and slightly by vehicle B (Fig. 16).

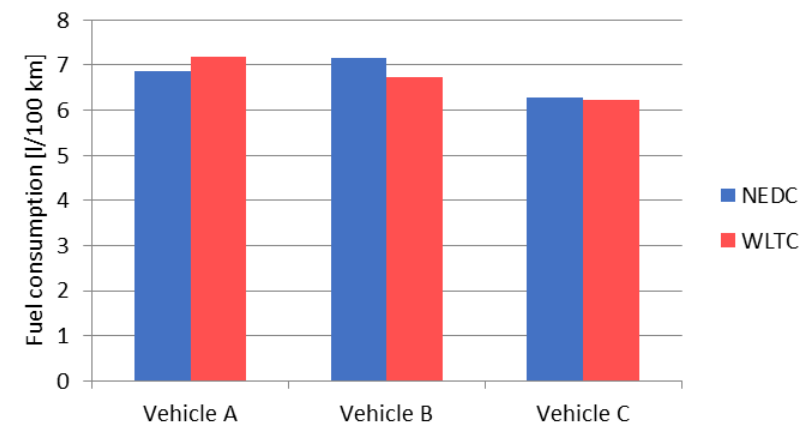

Fig. 12. Fuel consumption in NEDC and WLTC tests

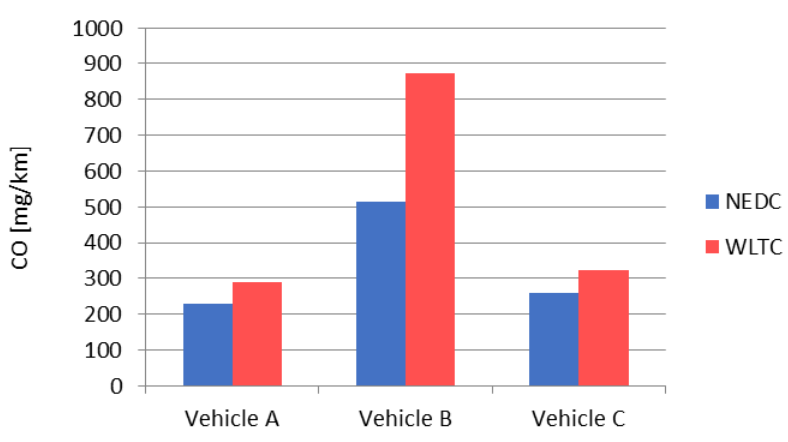

Fig. 13. Carbon monoxide emissions in NEDC and WLTC tests 


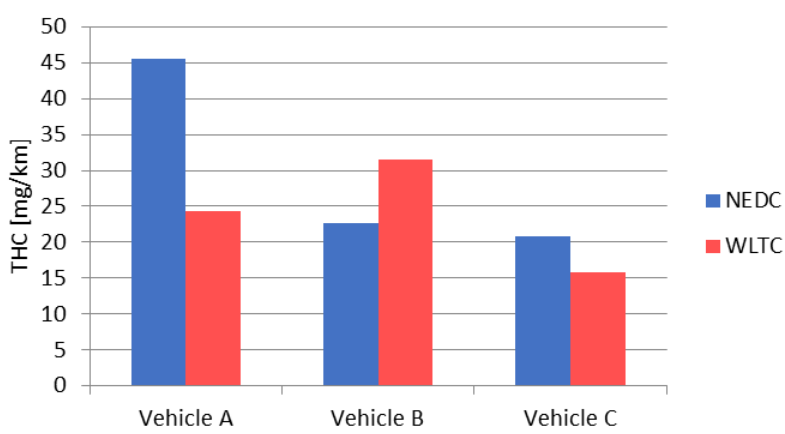

Fig. 14. Hydrocarbons emissions in NEDC and WLTC tests

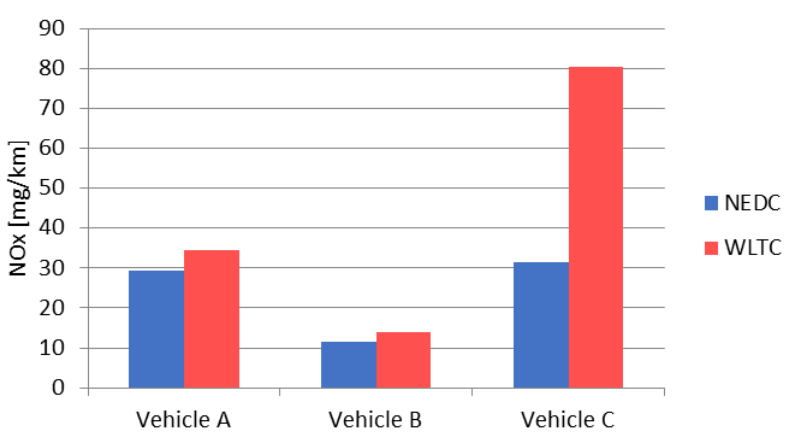

Fig. 15. Nitrogen oxides emissions in NEDC and WLTC tests

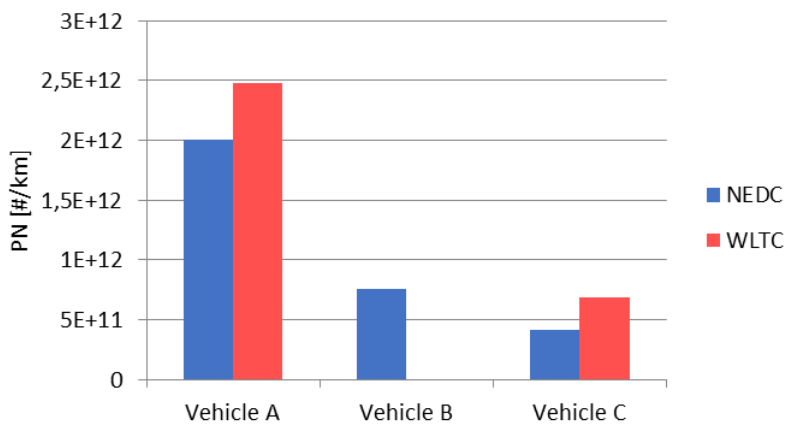

Fig. 16. Particle emissions in NEDC and WLTC tests

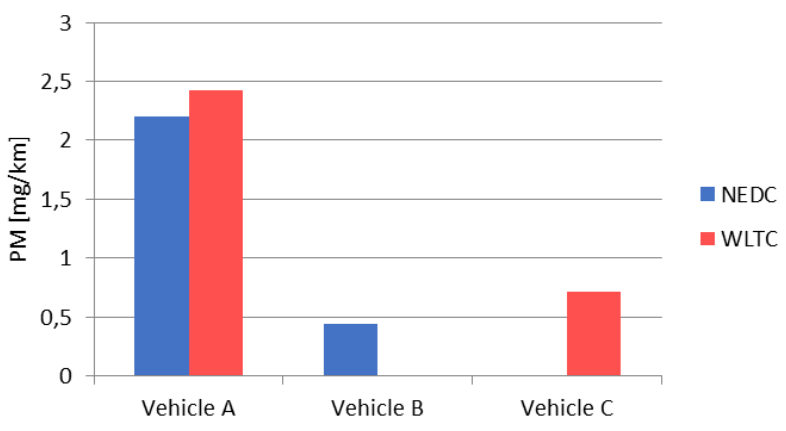

Fig. 17. Particle emissions in NEDC and WLTC tests

\section{Conclusions}

Measurements of fuel consumption and emissions of regulated exhaust compounds in 3 different passenger cars equipped with gasoline engines of the same displacement $1.4 \mathrm{dm}^{3}$ but different design solutions (direct and indirect injection, turbocharging and natural aspiration) were carried out. Each of the cars was tested on a chassis dynamometer in the conditions of two driving cycles: the New European Driving Cycle (NEDC) and the Worldwide Harmonized Light Duty Test Cycle (WLTC). In both cycles, a given tested car was equipped and configured in the same way.

The fuel consumption obtained in the new WLTC cycle only in the case of one of the cars tested was $4.5 \%$ higher than in the NEDC cycle. In the case of the second car it was lower by $6 \%$ and in the case of the third car the fuel consumption were practically the same in both cycles (smaller in WLTC by less than 1\%). This indicates that the introduction of a new driving cycle may not significantly increase the fuel consumption obtained in the tests. The introduction of the new Worldwide Harmonized Light Duty Test Procedure (WLTP), which relevant part is WLTC, was intended to bring the results obtained in type-approval tests closer to the results obtained in normal road operation. However, it should be emphasized that the WLTC driving cycle is just one of the factors that distinguishes the new WLTP procedure from the previous NEDC one. Other important factors affecting fuel consumption have not been the subject of this study (WLTP limits the possibilities of unrealistic reduction of vehicle weight, energy consumption by auxiliary equipment, resistance of movement, etc.).

For all cars tested $\mathrm{CO}, \mathrm{NO}_{\mathrm{x}}, \mathrm{PN}$ and $\mathrm{PM}$ emissions in the WLTC cycle were higher than in the NEDC cycle, on average for the three examined cars by: 48\%, 78\%, 30\% and $10 \%$ respectively.

The average for the three cars' THC emission in the WLTC cycle was lower by $20 \%$ compared to the emission in the NEDC cycle. However, in this case the impact of the cycle is ambiguous, because the lower THC emission was obtained for the two cars tested, and for one car the THC emission was higher in the WLTC cycle.

Summarising, it can be concluded that replacing the NEDC with WLTC Class 3 driving cycle itself does not result in higher fuel consumption, and so it does not contribute much to the elimination of the problem of significantly lower type-approval fuel consumption than the actual one. However, the emissions of regulated exhaust compounds determined in the new WLTC driving cycle are higher than emissions obtained in the NEDC cycle, except for THC.

\section{Nomenclature}

DI direct injection

EUDC extra-urban driving cycle

FC fuel consumption, $\mathrm{dm}^{3} / 100 \mathrm{~km}$

MPI multi-point injection

NEDC new European driving cycle

PM particulate matter
PN particle number

SI spark ignition

UDC urban driving cycle

WLTC worldwide harmonized light duty vehicle test cycle

WLTP worldwide harmonized light duty vehicl test procedure 


\section{Bibliography}

[1] BIELACZYC, P., SZCZOTKA, A., WOODBURN, J. Exhaust emissions of particulate matter from light-duty vehicles - an overview and the current situation. Combustion Engines. 2017, 171(4), 227-238. DOI: 10.19206/CE-2017439

[2] DUARTE, G.O., GONÇALVES, G.A., FARIAS, T.L. Analysis of fuel consumption and pollutant emissions of regulated and alternative driving cycles based on real-world measurements. Transportation Research Part D. 2016, 44, 43-54. DOI: 10.1016/j.trd.2016.02.009.

[3] JIMÉNEZA, J.L., VALIDOB, J., MOLDENC, N. The drivers behind differences between official and actual vehicle efficiency and $\mathrm{CO}_{2}$ emissions. Transportation Research Part D. 2019, 67, 628-641. DOI: 10.1016/j.trd.2019.01.016

[4] MERKISZ, J., PIELECHA, J. Selected remarks about RDE test. Combustion Engines. 2016, 166(3), 54-61. DOI: 10.19206/CE-2016-340

[5] PAVlOVIC, J., MAROTTA, A., CIUFFO, B. $\mathrm{CO}_{2}$ emissions and energy demands of vehicles tested under the NEDC and the new WLTP type approval test procedures. Applied Energy. 2016, 177, 661-670. DOI: 10.1016/ j.apenergy.2016.05.110

Grzegorz Koszałka, DSc., DEng. - Faculty of Mechanical Engineering, Lublin University of Techno$\log$.

e-mail: g.koszalka@pollub.pl
[6] TIETGE, U., ZACHAROF, N., MOCK, P. et al. From laboratory to road - a 2015 update of official and "real-world" fuel consumption and $\mathrm{CO}_{2}$ values for passenger cars in Europe. The International Council on Clean Transportation. 2015.

[7] TSIAKMAKIS, S., FONTARAS, G., ANAGNOSTOPOULOS, K. et al. A simulation based approach for quantifying $\mathrm{CO}_{2}$ emissions of light duty vehicle fleets. A case study on WLTP introduction. Transportation Research Procedia. 2017, 25, 3898-3908. DOI: 10.1016/j.trpro.2017.05.308

[8] VARELLA, R.A., FARIA, M.V., MENDOZA-VILLAFUERTE, P. et al. Assessing the influence of boundary conditions, driving behavior and data analysis methods on real driving $\mathrm{CO}_{2}$ and $\mathrm{NO}_{\mathrm{x}}$ emissions. Science of the Total Environment. 2019, 658, 879-894. DOI: 10.1016/j.scitotenv. 2018.12.053.

[9] ZACHAROF, N., TIETGE U., FRANCO, V., MOCK, P. Type approval and real-world $\mathrm{CO}_{2}$ and $\mathrm{NO}_{\mathrm{x}}$ emissions from EU light commercial vehicles. Energy Policy. 2016, 97, 540-548. DOI: 10.1016/j.enpol.2016.08.002

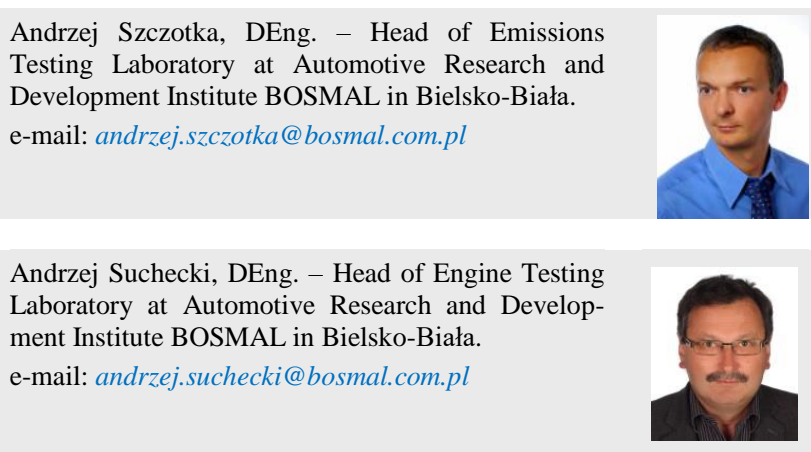

\author{
Development Institute BOSMAL in Bielsko-Biała. \\ -mail: andrzej.szczotka@bosmal.com.pl
}

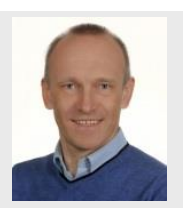

